\title{
Prevalencia de sífilis y características del comportamiento de los jóvenes indígenas del Paraguay, 2016
}

\author{
Analía Ortiz ${ }^{1,5}, *$ Gladys Estigarribia ${ }^{1}$, Gloria Aguilar ${ }^{1,2,4}$, Angélica Espinosa Miranda ${ }^{2}$, William Mc \\ Farland $^{3}$, Carlos Miguel Rios-González ${ }^{1,5}$, Anibal Kawabata ${ }^{4}$, Sergio Muñoz ${ }^{6}$, Tania Samudio ${ }^{4}$, Gladys \\ López ${ }^{4}$, Aldo Rodriguez ${ }^{1,5}$
}

\author{
${ }^{1}$ Universidad Nacional de Caaguazú. Instituto Regional de Investigación en Salud. Cnel. Oviedo, Paraguay \\ ${ }^{2}$ Universidad Federal del Espíritu Santo, Brasil \\ ${ }^{3}$ Universidad de California, Ciencias de la Salud Global. San Francisco, California, Estados Unidos \\ ${ }^{4}$ Ministerio de Salud Pública y Bienestar Social. Programa Nacional de Lucha Contra el Sida (PRONASIDA). \\ Paraguay \\ ${ }^{5}$ Universidad Nacional de Caaguazú, Facultad de Ciencias Médicas. Cnel. Oviedo, Paraguay \\ ${ }^{6}$ Universidad de la Frontera. Facultad de Medicina. Temuco, Chile
}

Cómo referenciar este artículo/ How to reference this article:
Ortiz A, Estigarribia G, Aguilar G, Espinosa Miranda A, Farland W, Ríos-González CM, et al. Prevalencia de sífilis y características del comportamiento de los jóvenes indígenas del Paraguay, 2016. Mem. Inst. Investig. Cienc. Salud. 2018; 16(3): 51-57

\section{R E S U M E N}

El objetivo fue determinar la prevalencia de sífilis y características de comportamiento de los jóvenes indígenas del Paraguay en el 2016. Se realizó un estudio observacional, descriptivo de corte transversal con muestro probabilístico estratificado bietapico, que incluyó a jóvenes indígenas de 15 a 18 años de cinco familias lingüísticas de Paraguay. Se utilizó un cuestionario estructurado y para el tamizaje de sífilis se utilizó una test rápido treponémico y para confirmar los resultados reactivos se realizó VDRL, considerando como resultado positivo si el test rápido era positivo + VDRL positivo a una dilución de 1:4 o mayor y si la VDRL era menor a 1:4 con TPHA positivo. Los resultados se expresan como medidas de tendencia central, dispersión y proporciones. Ingresaron al estudio 546 jóvenes de 15 a 18 años, el $67,03 \%$ era del sexo femenino. Se identificaron 36 casos de sífilis, que representa una prevalencia de 6,6\% [IC95\%: 4,7-9,0]. Mayor prevalencia de sífilis se observó en los jóvenes que consumieron alcohol en la última relación sexual 20,6\% (14/68) $(p<0,001)$, sexo transaccional $40 \%(2 / 5)(p=0,010)$ y no utilización de condón $20,6 \%$ $(14 / 68)(p<0,001)$. Se encontró una alta prevalencia de sífilis en la población juvenil indígena, relacionada al consumo de alcohol en la última relación, práctica de sexo transaccional y no utilización de preservativos. Se recomienda la implementación de estrategias específicas con abordaje intercultural apropiadas para una población joven, orientadas a disminuir las prácticas de riesgo y promocionar la utilización de preservativos.

Palabras clave: Enfermedades de Transmisión Sexual; Salud de Poblaciones Indígenas; Epidemiologia.

\section{Prevalence of syphilis and characteristics of the behavior of indigenous young people of Paraguay, 2016}

\section{A B S T R A C T}

The objective was to determine the prevalence of syphilis and behavioral characteristics of indigenous youth of Paraguay in 2016. An observational, descriptive, cross-sectional study was carried out with multistage stratified probabilistic sampling. Indigenous youth aged 15 to 18 from five linguistic families living in Paraguay were included. A structured questionnaire was used and a rapid treponemal test was used for the syphilis screening. The results are expressed in measures of central tendency, dispersion and proportions. Five 
hundred forty six young people from 15 to 18 years old entered the study, $67.03 \%$ was female. There were 36 cases of syphilis detected, resulting in a prevalence of $6.6 \%$ [ $95 \%$ $\mathrm{CI}$ : 4.7-9.0]. Young people who consumed alcohol in the last sexual intercourse $20.6 \%$ $(14 / 68)(p=0.000)$, transactional sex $40 \%(2 / 5)(p=0.010)$ and non-use of condom $20.6 \%(14 / 68)(p=0.000)$ had higher prevalence of syphilis. We found a high prevalence of syphilis in the indigenous juvenile population related to drinking alcohol during the last relationship, practice of transactional sex and the non-use of condoms. Specific strategies should be implemented with an intercultural approach appropriate for a young population, aimed at reducing risk practices and promoting the use of condoms.

Keywords: sexually transmitted diseases, health of indigenous people, epidemiology.

\section{INTRODUCCIÓN}

Según los datos del Centro para el Control y Prevención de Enfermedades, en el 2012 se estimaron 18 millones de casos de sífilis en todo el mundo, la mayoría de los cuales se encontraban en países de ingresos bajos y medios; a su vez durante ese mismo año se estimó una prevalencia de 0,5\% en la población entre 15 y 49 años. En la actualidad aún se considera a la sífilis como un importante problema para la salud pública mundial ${ }^{(1)}$. La incidencia anual de casos de sífilis en la población adulta se ha estimado en 3 millones para Latinoamérica y el Caribe ${ }^{(2)}$.

La situación epidemiológica de la sífilis en Latinoamérica es variable. En Brasil a partir de un estudio en 36.173 parturientas en el 2011 se estimó la prevalencia global de sífilis para el país en $0,89 \%$ mientras que en las regiones Norte, Nordeste, Sudeste, Sur y Centro oeste fue de $1,05 \%, 1,14 \%, 0,73 \%, 0,48 \%$ y $1,20 \%$, respectivamente. Los datos actualizados del 2016 revelan que fueron notificados en el Brasil 87.593 casos de sífilis adquirida, 37.436 casos de sífilis en embarazadas y 20.474 casos de sífilis congénita, entre ellos 185 óbitos $^{(3)}$.

Paraguay no se encuentra ajeno a esta problemática, si bien la prevalencia varía según las distintas poblaciones estudiadas, siendo mayor en las personas trans $(20 \%)$, seguido de hombres que tienen sexo con hombres (HSH) (10\%), mujeres trabajadoras sexuales (MTS) $(9,8 \%)$; mientras que en las puérperas y parturientas la prevalencia fue de $4.41 \%$ en el 2012 y en la población indígena fue de $9,7 \%$ en el $2011^{(4)}$. La salud de la población indígena se encuentra enmarcada en el estigma del colonialismo que aún persiste hasta hoy en día. La vulnerabilidad y la dificultad del acceso a la salud actúan como condicionantes para el aumento en las tasas de las infecciones de transmisión sexual (ITS), como lo es la sífilis, lo cual predispone a desenlaces desfavorables para la salud sexual y reproductiva ${ }^{(5)}$.

Los adolescentes se identifican como una población de riesgo ya que no solo realizan actividades sexuales sin protección con mayor frecuencia, sino que también se descubre que estos tienen múltiples parejas sexuales, practican la prostitución, sufren de explotación sexual y tienen dificultades con recursos tales como transporte para visitas médicas, es poco probable que hablen abiertamente acerca de tener relaciones sexuales, lo que inevitablemente afecta la frecuencia de pruebas y tratamiento de $\operatorname{ITS}^{(6)}$. Se estima que existe una falta de confianza entre los indígenas para acudir a los servicios de salud, que va en aumento entre las comunidades indígenas vinculado con la falta de medidas adecuadas. Numerosas prácticas, asociado a las condiciones de vida y al poco conocimiento sobre estas enfermedades, debido a la dificultad de acceso a información práctica para su salud, condicionan que dicha población sea vulnerable para esta infección ${ }^{(7)}$.

Con base en lo anterior, se planteó como objetivo determinar la prevalencia de sífilis y las características de comportamiento de los jóvenes indígenas del Paraguay durante el 2016.

\section{MATERIAL Y MÉTODOS}

Se realizó un estudio observacional, descriptivo, de corte transversal, con componente analítico, con muestreo probabilístico estratificado bietapico sobre la prevalencia de sífilis y características del comportamiento de los jóvenes indígenas del Paraguay durante el 2016.

Se incluyeron en el estudio a jóvenes indígenas de 15 a 18 años de ambos sexos, de cinco familias lingüísticas (Zamuco, Mataco Mataguayo, Lengua Maskoi, Guaicurú, Guaraní) residentes en Paraguay en el momento del estudio. Fueron reclutados mediantes un muestreo probabilístico estratificado bietapico: a) la primera etapa se estratificó el país por región geográfica y familia lingüística, estableciéndose 10 estratos, y b) la segunda una selección aleatoria de las comunidades dentro de los estratos. Para la determinación del 
tamaño muestral se utilizó la información disponible en la Dirección General de Encuestas, Estadísticas y Censos y el Tercer Censo Nacional de población y hogar para pueblos indígenas $^{(8)}$, considerándose los siguientes parámetros, tamaño de la población: 9.777 indígenas de 15 a 18 años, proporción esperada: 6, precisión: 1.8, IC 95\%, arrojando un tamaño de la muestra de 472 sujetos.

Para la recolección de los datos, se procedió a la realizar visitas y reuniones con los líderes de las comunidades seleccionadas, estas consultas consistieron en contextualización y solicitud de permiso para acceder a dichas comunidades, en todo momento se contó con el apoyo de un consultor de interculturalidad para garantizar el enfoque apropiado de la cosmovisión indígena y buscar el consentimiento previo libre e informado, a su vez se contó con la participación de una persona bilingüe que manejó el idioma de las familias lingüísticas y el español para el cumplimiento de la encuesta. Los encuestadores explicaron en un lenguaje claro y amigable los objetivos y el alcance de la investigación y solicitaron el consentimiento informado de los padres o tutores, y el asentimiento del menor.

Las entrevistas se realizaron utilizando un instrumento que se basó en las directrices para encuestas de comportamiento repetido en poblaciones con riesgo de VIH adaptadas de Family Health International, la cual estaba compuesta por 44 preguntas estructuradas, divididas en 5 secciones (Identificación de la encuesta, información general, conocimiento de la prevención de la transmisión del VIH, historia sexual y otros factores de riesgo $)^{(9)}$.

El testeo de laboratorio fue realizado en la comunidad indígena. Las muestras de sangre para confirmación del diagnóstico de sífilis y control de calidad fueron conservadas de 2 a 8 grados para ser transportadas al laboratorio de referencia del PRONASIDA. El test rápido para anticuerpos para la sífilis fue realizado utilizando la prueba treponemica (Advanced Quality, China) para screening y si era reactivo, posteriormente se realizó la Venereal Dissease Research Laboratory (Wiener, Argentina) para detectar infección activa de sífilis, considerada como Test rápido positivo+VDRL dilución igual o mayor a $1: 4$ y si la VDRL era menor a 1:4 con TPHA positivo.

Este estudio fue aprobado por el Comité de Investigación Ética, del Instituto de Medicina Tropical bajo el protocolo Ref.: 117/2016. Todas las etapas de la investigación responden a los principios éticos de confidencialidad, igualdad y justicia. Todos los casos positivos se derivaron a PRONASIDA y sus dependencias para el tratamiento y seguimiento correspondientes.

Se utilizó el software estadístico STATA 14.0 ® (Stata Corporation, College Station, Texas, EEUU). Se realizó un primer análisis univariado y luego bivariado. El test exacto de Fisher fue utilizado para analizar las diferencias entre las proporciones. Los valores con $\mathrm{p}<0,05$ fueron considerados estadísticamente significativos.

\section{RESULTADOS}

Ingresaron al estudio 546 jóvenes de 15 a 18 años, el $67.03 \%(366 / 546)$ era del sexo femenino, el $71.43 \%(390 / 546)$ residía en la región oriental, el $65.02 \%(355 / 546)$ correspondía a la familia lingüística guaraní y el $88.46 \%$ tuvo acceso a educación (483/546) (Tabla 1).

Tabla 1: Características demográficas de jóvenes indígenas del Paraguay. 2017. $\mathrm{N}=546$

\begin{tabular}{lll}
\hline Características & $\mathbf{n}$ & \% \\
\hline Sexo & & \\
$\quad$ Masculino & 180 & 32.97 \\
$\quad$ Femenino & 366 & 67.03 \\
Región de residencia & & \\
$\quad$ Oriental & 390 & 71.43 \\
$\quad$ Occidental & 156 & 28.57 \\
Familias lingüísticas & & \\
$\quad$ Zamuco & 8 & 1.47 \\
\hline
\end{tabular}




\begin{tabular}{lll}
\hline Mataco & 75 & 13.74 \\
Lengua Maskoi & 85 & 15.57 \\
Guaicurú & 23 & 4.21 \\
Guaraní & 355 & 65.02 \\
Acceso a educación & & \\
Si & 483 & 88.46 \\
No & 63 & 11.54 \\
\hline
\end{tabular}

Se detectaron 36 casos de sífilis de los 546 jóvenes indígenas, resultando en una prevalencia de 6.6\% (IC 95\%: 4.7-9.0) (Tabla 2). La prevalencia de sífilis en hombres fue del $7.8 \%(4.3-12.7)$ y en mujeres $6.0 \%(3.8-9.0)$. La prevalencia en la región Oriental fue de (6.7\% [IC 95\%: 4.4-9.6]) (26/390). Considerando las familias lingüísticas, la prevalencia en la familia Mataco fue de (12.0\% [IC 95\%: 5.6-21.6]) (9/75), Guaraníes (7.0\% [IC 95\%: 4.6-10.2]) (25/355), Guaicurú (4.4\% [IC 95\%: 0.11-21.9]) (1/23), Lengua Maskoi (1.2\% [IC 95\%: 0.0-6.4]) (1/85) y Zamuco no presentó casos de sífilis. La prevalencia de sífilis en los que tuvieron acceso a educación, fue del (6.8\% [IC 95\%: 1.013.2]) (33/483) (Tabla 2).

Tabla 2: Prevalencia de sífilis de jóvenes indígenas del Paraguay. $n=546$

\begin{tabular}{llll}
\hline & \multicolumn{2}{l}{ Prevalencia de sífilis } & \\
\hline $\begin{array}{l}\text { Características } \\
\text { Sexo }\end{array}$ & $\mathrm{n} / \mathrm{N}$ & $\%(95 \% \mathrm{IC})$ & Valor $p$ \\
$\quad$ Masculino & & & \\
$\quad$ Femenino & $14 / 180$ & $7.8(4.3-12.7)$ & 0.434 \\
Región de residencia & $22 / 366$ & $6.0(3.8-9.0)$ & \\
$\quad$ Oriental & & & 0.913 \\
$\quad$ Occidental & $26 / 390$ & $6.7(4.4-9.6)$ & \\
Familia lingüística & $10 / 156$ & $6.4(3.1-11.5)$ & 0.076 \\
$\quad$ Zamuco & & & \\
$\quad$ Mataco & $0 / 8$ & $0.0(0.0-0.0)$ & \\
$\quad$ Lengua Maskoi & $9 / 75$. & $12.0(5.6-21.6)$ & \\
$\quad$ Guaicurú & $1 / 85$. & $1.2(0.0-6.4)$ & \\
$\quad$ Guaraní & $1 / 23$. & $4.4(0.11-21.9)$ & \\
Acceso a educación & $25 / 355$ & $7.0(4.6-10.2)$ & \\
$\quad$ Si & $33 / 483$ & $6.8(1.0-13.2)$ & \\
$\quad$ No & $3 / 63$. & $4.8(4.7-9.5)$ & \\
$\quad$ Total Prevalencia & $36 / 546$ & $6.59(4.7-9.0)$ & \\
\hline
\end{tabular}

Los jóvenes que presentaron mayor prevalencia de sífilis fueron los que consumieron alcohol en la última relación $20.6 \%(14 / 68)(p<0,001)$, practicaban sexo transaccional $40 \%$ $(2 / 5)(p=0,010)$ y no utilizaban condón $20.6 \%(14 / 68)(p<0,001)$, (Tabla 3$)$. 
Tabla 3: Factores asociados a sífilis en jóvenes indígenas sexualmente activos del Paraguay 2017. $\mathrm{N}=380$

\begin{tabular}{|c|c|c|c|}
\hline & $\begin{array}{l}\text { Prevalencia de } \\
\text { sífilis }(n / N)\end{array}$ & $\%(95 \%$ CI $)$ & p-value \\
\hline \multicolumn{4}{|l|}{ Edad de inicio de relación sexual } \\
\hline$<15$ años & $17 / 210$ & $8,1(4,8-12,6)$ & 0,799 \\
\hline$\geq 15$ años & $15 / 170$ & $8,8(5,0-14,1)$ & \\
\hline \multicolumn{4}{|l|}{$\begin{array}{l}\text { Número de parejas en el último } \\
\text { año }\end{array}$} \\
\hline$\leq 1$ & $18 / 254$ & $7,1(4,3-11,0)$ & 0,184 \\
\hline$>1$ & $14 / 126$ & $11,1(6,2-17,9)$ & \\
\hline \multicolumn{4}{|l|}{ Parejas sexuales en el último mes } \\
\hline$\leq 1$ & $28 / 328$ & $8.5(5.7-12.1)$ & 0,415 \\
\hline$>1$ & 4/31, & $12,9(3,6-30,0)$ & \\
\hline \multicolumn{4}{|l|}{$\begin{array}{l}\text { Uso de alcohol en la última } \\
\text { relación }\end{array}$} \\
\hline Si & $14 / 68$ & $20,6(11,7-32,1)$ & $<.001$ \\
\hline No & $18 / 302$ & $5,9(3,6-9,3)$ & \\
\hline \multicolumn{4}{|l|}{ Sexo transaccional } \\
\hline Si & $2 / 5$ & $40(5,3-85,3)$ & 0.010 \\
\hline & $29 / 365$ & $7,9(5,4-11,2)$ & \\
\hline \multicolumn{4}{|c|}{$\begin{array}{l}\text { Uso de condón en la última } \\
\text { relación }\end{array}$} \\
\hline $\mathrm{Si}$ & $18 / 302$ & $6,0(3,6,-9,3)$ & $<0.001$ \\
\hline No & $14 / 68$ & $20,6(11,73-32,1)$ & \\
\hline \multicolumn{4}{|l|}{ Pareja estable } \\
\hline Si & $25 / 267$ & $9,4(6,2-13,5)$ & 0.291 \\
\hline No & $6 / 101$ & $5,9(2,2-12,5)$ & \\
\hline
\end{tabular}

\section{DISCUSIÓN}

Garantizar el acceso a la atención primaria de salud es ampliamente aceptado como clave para mejorar los resultados de salud. En el caso de las poblaciones indígenas que viven con altas tasas de enfermedades infectocontagiosas, el acceso a estos servicios es aún más crucial. Estudios que traten exclusivamente las tasas de prevalencia en la población masculina no se encuentran disponibles, sin embargo, estudios que abordan la prevalencia en las embarazadas están ampliamente difundidos, lo cual es entendible por el impacto que presenta durante el embarazo, tales como aborto espontáneo, malformaciones, entre otros ${ }^{(10)}$.

La prevalencia de sífilis encontrada en jóvenes indígenas de cinco familias lingüísticas del Paraguay fue alta en comparación a la prevalencia registrada en el país ${ }^{(4)}$, esta variación de la prevalencia se pudo apreciar de acuerdo a las familias, registrándose mayoritariamente en la familia Mataco, Guaraníes y Guaicurúes, si bien es importante considerar la proporción de sujetos en cada comunidad indígena, siendo la mayor densidad poblacional la de los Guaraníes $^{(8)}$, por lo que la prevalencia registrada en esta población no es despreciable, por su amplia distribución en todo el territorio nacional.

Las publicaciones en esta área son escasas ${ }^{(11)}$, por lo que la comparación de los grupo resulta difícil, sin embargo, en comparación con los resultados de Benzaken et al., 2017(3), Orellana ER et al., 2013(12) y Bartlett E et al., 2008 ${ }^{(13)}$ la prevalencia ha sido mayor en este estudio, asimismo respecto al sexo, al igual que los estudios anteriores se pudo apreciar que la mayor prevalencia se ha encontrado en el sexo masculino ${ }^{(3,12,13)}$. En comparación con la población no indígena se encuentra 2 veces más elevada con relación a los datos

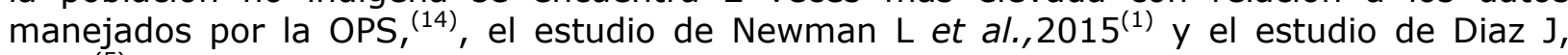
$2013^{(5)}$.

En este estudio también se pudo apreciar la importante proporción de participantes que ha involucrado alcohol durante las prácticas sexuales. En este punto, existen similitudes con 
estudios llevado a cabo por Zoni et al., 2013(15), Jalil EM et al.,(16) y Kakchapati $S$ et al., $2017^{(17)}$, que si bien no son estudios llevados a cabo en población indígena, demuestran el consumo de alcohol como práctica de riesgo para adquisición de ITS.

Las limitaciones del estudio se centran en el sesgo de memoria, puesto que al ser consultados sobre las prácticas de riesgo, es posible que algunos de los participantes no hayan revelado la verdadera practica realizada.

El abordaje de los determinantes sociales y económicos más amplios de la vulnerabilidad a las ITS es una gran tarea para la salud pública del país. Los desequilibrios profundamente arraigados de ese tipo no se pueden remediar fácil o rápidamente, es por ello que la estrategias educativas adaptadas culturalmente y de monitoreo constante son de vital importancia para disminuir las tasa de infección en esta población.

A modo de conclusión, existe una alta prevalencia de sífilis en la población juvenil indígena, tres veces mayor que el promedio en Latinoamérica y unas dos veces más que la población general de Paraguay.

Financiación: El proyecto de investigación fue financiado por el Programa Paraguayo para el Desarrollo de la Ciencia y Tecnología (PROCIENCIA) del Consejo Nacional de Ciencia y Tecnología (CONACYT)

Los autores no declaran conflicto de intereses.

\section{REFERENCIAS BIBLIOGRAFÍCAS}

1. Newman L, Rowley J, Vander Hoorn S, Wijesooriya NS, Unemo M, Low N, et al. Global Estimates of the Prevalence and Incidence of Four Curable Sexually Transmitted Infections in 2012 Based on Systematic Review and Global Reporting. PLoS One. 2015;10(12):e0143304.

2. Centros para el Control y Prevención de Enfermedades. Sífilis [Internet]. [citado 30 de Mayo de 2016]. Disponible en: https://www.cdc.gov/std/spanish/sifilis/def ault.htm

3. Benzaken AS, Sabidó M, Brito I, Bermúdez $X P D$, Benzaken NS, Galbán E, et al. HIV and syphilis in the context of community vulnerability among indigenous people in the Brazilian Amazon. Int J Equity Health. 2017;16(1):92.

4. Ministerio de Salud Pública y Bienestar Social. Informe Situación Epidemiológica del VIH Paraguay 2016 [Internet]. 2016 [citado 20 de Marzo de 2018]. Disponible en:

http://www.pronasida.gov.py/images/docu mentos/inf.epi.vih.2016.pdf

5. Díaz J. Vigilancia epidemiológica de sífilis y gonorrea. Rev Chil infectología. 2013;30(3):303-10.

6. Garcés JP, Rubiano LC, Orobio $Y$, Castaño M, Benavides E, Cruz A. La educación del personal de salud: clave para la eliminación de la sífilis congénita en Colombia. Biomédica. 2017;37(3):416-24.

7. Burroughs A, Guy R, Su J-Y, Giele C, Hellard $M$, Kong $M$, et al. Recent trends in syphilis in aboriginal and Torres strait islander people and non-indigenous persons in Australia; an analysis of routine surveillance data. [citado 29 de Mayo de 2018]; Disponible en: https://www.eiseverywhere.com/file_uploa ds/40b54145f46f8ef014a3868de9e2add8_ 195_AmyBurroughs.pdf

8. Dirección de Estadísticas Sociales y Demográficas. III Censo Nacional de Población y Viviendas para pueblos Indigenas,2012 [Internet]. 2012 [Citado 30 de Mayo de 2018]. Disponible en: http://www.dgeec.gov.py/Publicaciones/Bi blioteca/censo indigena 2012/Pueblos indigenas en el Paraguay Resultados preliminares - CNI 2012.pdf

9. Amon J, Brown T, Hogle J, Macneil J, Magnani R, Mills S, et al. Encuesta de Vigilancia del Comportamiento: Directrices para encuestas del comportamiento repetidas en población en riesgo al VIH [Internet]. [Citado 30 de Mayo de 2018]. Disponible en: http://www.who.int/hiv/strategic/en/bss_f hi_sp.pdf

10. Fitzpatrick EFM, Martiniuk ALC, D'Antoine H, Oscar J, Carter M, Elliott EJ. Seeking consent for research with indigenous communities: a systematic review. BMC Med Ethics. 2016;17(1):1-18.

11. Rios-González CM. Evaluation of the scientific production on HIV in indigenous people, from 1989 to 2016. Travel Med Infect Dis. 2017 Jul - Aug;18:83-4. doi: 10.1016/j.tmaid.2017.06.004.

12. Orellana ER, Alva IE, Cárcamo CP, García PJ. Structural factors that increase HIV/STI vulnerability among indigenous people in the Peruvian amazon. Qual Health Res. 2013;23(9):1240-50.

13. Bartlett EC, Zavaleta C, Fernández C, Razuri $\mathrm{H}$, Vilcarromero $\mathrm{S}$, Vermund $\mathrm{SH}$, et al. Expansion of HIV and syphilis into the Peruvian Amazon: a survey of four communities of an indigenous Amazonian 
ethnic group. Int J Infect Dis. 2008; 12(6): e89-94.

14. OPS. OPS/OMS Paraguay - Metas asistenciales: Sífilis, VIH y Mujeres en gestación [Internet]. [Citado 30 de Mayo de 2018]. Disponible en: https://www.paho.org/par/index.php?optio $\mathrm{n}=$ com_content\&view $=$ article\&id=270: met as-asistenciales-sifilis-vih-mujeresgestacion\&Itemid $=255$

15. Zoni AC, González MA, Sjögren HW. Syphilis in the most at-risk populations in Latin America and the Caribbean: a systematic review. Int $\mathrm{J}$ Infect Dis. 2013;17(2):e84-92.
16. Jalil EM, Wilson EC, Luz PM, Velasque $L$, Moreira RI, Castro C V., et al. HIV testing and the care continuum among transgender women: population estimates from Rio de Janeiro, Brazil. J Int AIDS Soc. 2017;20(1):21873.

17. Kakchapati $S$, Paudel $T$, Maharjan $M$, Lim A. Systematic Differences in HIV, Syphilis and Risk Behaviors among Street Based and Establishment Based Female Sex Workers in Kathmandu Valley of Nepal. Nepal J Epidemiol. 2017;6(4): 62030. 